\title{
Using Hard Classifiers to Estimate Conditional Class Probabilities
}

\author{
Ole Martin Halck \\ Norwegian Defence Research Establishment (FFI) \\ P.O. Box 25, NO-2027 Kjeller, Norway \\ ole-martin.halck@ffi.no
}

\begin{abstract}
In many classification problems, it is desirable to have estimates of conditional class probabilities rather than just "hard" class predictions. Many algorithms specifically designed for this purpose exist; here, we present a way in which hard classification algorithms may be applied to this problem without modification. The main idea is that by stochastically changing the class labels in the training data in a simple way, a classification algorithm may be used for estimating any contour of the conditional class probability function. The method has been tested on a toy problem and a problem with real-world data; both experiments yielded encouraging results.
\end{abstract}

\section{Introduction}

Classification is one of the most studied problems in machine learning research. In the simplest case, the task is to use training data to infer a classifier function $c: X \rightarrow\{0,1\}$, where $X$ is the instance or input space from which data points are taken. The usual measure of the quality of a classifier is the proportion of correct classifications it yields when given data not present in the training set. In the following, a classifier that outputs this kind of binary-valued predictions will be called a hard classifier.

Hard classifiers are clearly most useful when there is reason to believe that there is a deterministic relationship between each data point's position in input space and its class. In many problems this is not the case; this may be because all relevant information is not encoded in the input representation, or it may be due to real randomness in the problem domain. In such cases, it is desirable to have an indication of the certainty that a data point belongs to a given class. Learning machines that estimate actual probabilities of class membership of input data are particularly useful in this respect, for instance in problems where the costs of misclassification of positive and negative examples are different.. Soft two-class classification algorithms of this kind are essentially regression algorithms where a Bernoulli probability model is assumed.

Many methods have been devised for the estimation of conditional class probability functions, ranging from classical logistic regression, via neural networks 
and Bayesian methods (e.g. [1]), to recent advances in the field of kernel methods and support vector machines (SVMs) (e.g. [2,3,4,5]). In the latter area, we shall return to the work of Platt [2], who proposed fitting a logistic function to the unthresholded output of an SVM classifier, and of Wahba [3,4], who modified the cost function of a kernel method to obtain probabilistic outputs.

These previous approaches have in common that they require implementation (or modification) of methods specifically for conditional class probability estimation. In this paper, we present a novel approach where hard classifiers are used for addressing this task. In our method, only the training data need to be modified - in a simple way - while existing (hard) classification software can be used as-is.

Our approach is motivated and presented in Section 2 and subjected to experimental evaluation in Section 3. Section 4 discusses some theoretical and practical issues relating to this method, while Section 5 concludes the paper.

\section{Problem and Algorithm Formulation}

In this section, we formulate the problem of estimating conditional class probabilities, and relate it to the pure classification problem. This serves as a motivation for our algorithm, which is subsequently described. The presentation is kept at a somewhat informal level; a more thorough examination of the simplifications and assumptions made is given in Section 4.

\subsection{Conditional Class Probabilities and Hard Classifiers}

In the problem we study here, we assume that each data point $\mathbf{x}$ is drawn from an input space $X$ according to the probability distribution $p(\mathbf{x})$ and assigned to class $y=1$ with probability $p(y=1 \mid \mathbf{x})$ and to class $y=0$ otherwise. The task is to estimate the true conditional class probability $p(y=1 \mid \mathbf{x})$ as a function of $\mathbf{x}$, based on training data $\left\{\left(\mathbf{x}_{i}, y_{i}\right)\right\}_{i=1}^{L}$.

What is the relation between hard classification and this task? The goal in hard classification, assuming equal misclassification costs, is usually to obtain a function $c: X \rightarrow\{0,1\}$ that minimizes the expected classification error on unseen data. For a given data point $\mathbf{x}$, the expected error is minimized if the equivalence $c(\mathbf{x})=1 \Leftrightarrow p(y=1 \mid \mathbf{x})>\frac{1}{2}$ holds. Thus, the classification boundary of a hard classifier can be seen as an estimate of the surface in input space where $p(y=1 \mid \mathbf{x})=\frac{1}{2}$.

\subsection{Motivation for Our Algorithm}

The main idea of our algorithm is motivated by the simple relationship described above. There, the boundary $p(y=1 \mid \mathbf{x})=\frac{1}{2}$ is considered; clearly, if we can find the 
boundaries $p(y=1 \mid \mathbf{x})=q$ for other values of $q$ as well, we are able to estimate $p(y=1 \mid \mathbf{x})$ for a new example $\mathbf{x}$, by checking its place in the instance space in relation to the probability contours for the various values of $q$. The idea of our algorithm is to "shift" these contours by modifying the training data, so that the surface at which $p(y=1 \mid \mathbf{x})=q$ in the original data corresponds to the surface $p(y=1 \mid \mathbf{x})=\frac{1}{2}$ for the new data set. A hard classifier estimating the boundary $p(y=1 \mid \mathbf{x})=\frac{1}{2}$ can then be trained on this data set, and thus give an estimate of the contour $p(y=1 \mid \mathbf{x})=q$ in the original data set.

Specifically, consider the (unknown) surface in input space where $p(y=1 \mid \mathbf{x})$ has a given constant value $q<\frac{1}{2}$. Loosely stated, we may then expect that in our training set, a larger proportion than $q$ of the data points on one side of this surface belong to class 1, while this is true of less than a proportion $q$ of the points on the other side.

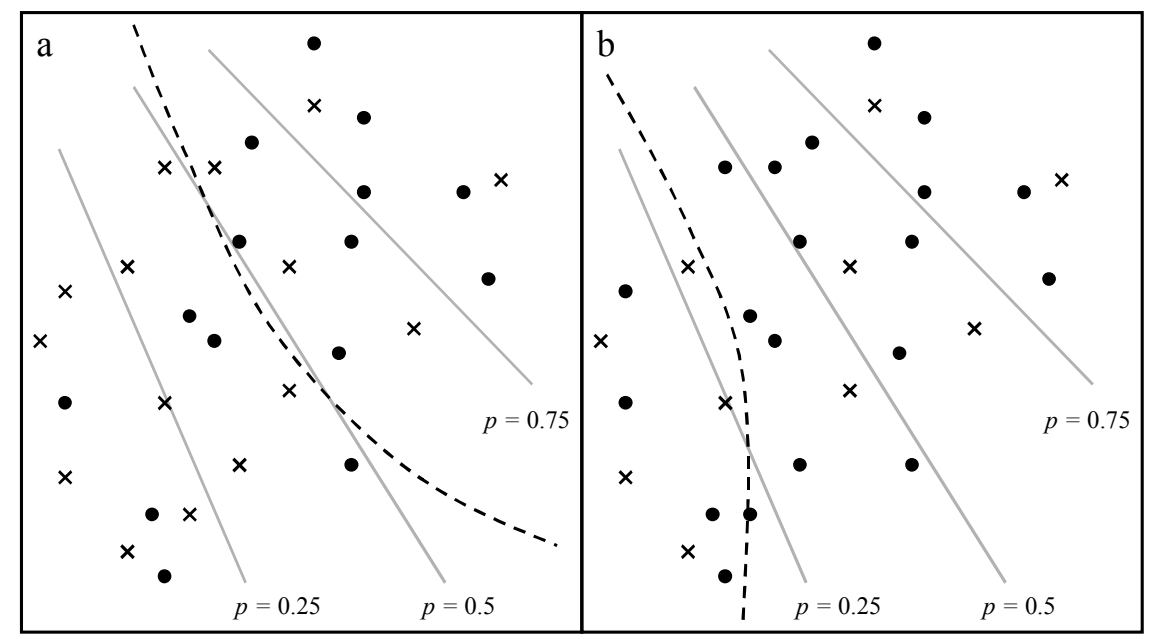

Fig. 1. Illustration of the idea of the algorithm. Training data are shown by dots (positive examples) and crosses (negative examples). The grey lines show contours of the true probability function; the dashed lines illustrate how a classifier might draw its decision boundary. a) Original training data; the decision boundary is an estimate of the 0.5 contour. b) Negative examples have been flipped to positives with a probability of $1 / 3$; the decision boundary now estimates the 0.25 contour

Now, assume that we create a new data set from the training set, where $\mathbf{x}$ is unchanged for each data point $(\mathbf{x}, y)$, while the class $y$ is set to 1 with probability $s=\left(\frac{1}{2}-q\right) /(1-q)$ regardless of its actual class, and otherwise left unchanged. In a region of input space where the original data set contains a proportion $r$ of positive examples, we may now expect that a proportion of approximately $r+(1-r) s=(r+1-2 q) / 2(1-q)$ of the modified data points are positive. This 
quantity clearly grows monotonously with $r$, and is equal to $\frac{1}{2}$ if and only if $r=q$. Thus, the decision boundary of a hard classifier trained on this modified data set may serve as an estimate of the $p(y=1 \mid \mathbf{x})=q$ contour surface in input space. Figure 1 illustrates this relationship in the case when $q=\frac{1}{4}$. For $q>\frac{1}{2}$, a similar operation, where the class labels are set to 0 with a suitable probability, may of course be performed.

In this way, we may estimate any contour surface $0<p(y=1 \mid \mathbf{x})<1$, and by building a collection of estimates of this kind we are able to approximate $p(y=1 \mid \mathbf{x})$ by a discrete function having the resolution we require. The following section describes this method in more detail, along with practical solutions to some problems that arise.

\subsection{Description of the Algorithm}

Given a set $D=\left\{d_{i}\right\}_{i=1}^{L}$ of training data, where $d_{i}=\left(\mathbf{x}_{i}, y_{i}\right)$, the first steps in using hard classifiers to form an estimate of the conditional class probability function $p(y=1 \mid \mathbf{x})$ are as follows:

1. Choose the coarseness of the estimating function. For simplicity, we divide the interval $(0,1)$ into $K$ equally-sized parts at the points $\left\{q_{k}\right\}_{k=1}^{-}$, where $q_{k}=k / K$ for $k=0,1, \ldots, K$.

2. For each $k$ from 1 to $K-1$, estimate the contour surface $p(y=1 \mid \mathbf{x})=q_{k}$ :

- $\quad$ Make a new data set $D^{(\kappa)}=\left\{\left(\mathbf{x}_{i}^{(\kappa)}, y_{i}^{(\kappa)}\right)\right\}_{i=1}^{L}$ from $D$ as follows: For each $i$ from 1 to $L$,

- $\quad$ set $\mathbf{x}_{i}^{(\kappa)} \leftarrow \mathbf{x}_{i}$;

- $\quad$ if $q_{k}<\frac{1}{2}$, set $y_{i}^{(n)} \leftarrow 1$ with probability $\left(\frac{1}{2}-q_{k}\right) /\left(1-q_{k}\right)$ and $y_{i}^{(n)} \leftarrow y_{i}$ otherwise;

- if $q_{k}>\frac{1}{2}$, set $y_{i}^{(\kappa)} \leftarrow 0$ with probability $\left(q_{k}-\frac{1}{2}\right) / q_{k}$ and $y_{i}^{(\kappa)} \leftarrow y_{i}$ otherwise.

- Train a hard classifier $c_{k}$ on the data set $D^{(k)}$.

Seen in isolation, the interpretation of a single classifier $c_{k}$ classifying a data point $\mathbf{x}$ as positive is that $p(y=1 \mid \mathbf{x})$ is estimated to be larger than $q_{k}$. A seemingly obvious way of obtaining a full estimate for $p(y=1 \mid \mathbf{x})$ is therefore to find $k$ such that $c_{i}(\mathbf{x})=1$ for $i \leq k$ and $c_{i}(\mathbf{x})=0$ for $i>k$, and use $\left(q_{k}+q_{k+1}\right) / 2$ as our estimate. Unfortunately, due to the stochasticity of the algorithm, it may (and does) happen that a classifier $c_{k}$ classifies a point as negative, while another classifier $c_{k^{\prime}>k}$ does not. To address this problem, we use a simple remedy that reduces to the procedure above when the outputs of the classifiers are indeed consistent with each other. The final step in the estimation of the conditional class probability function is then:

3. Estimate $p(y=1 \mid \mathbf{x})$ by 


$$
\hat{p}_{H C}(y=1 \mid \mathbf{x})=\left(\frac{1}{K} \sum_{k=1}^{K-1} c_{k}(\mathbf{x})\right)+\frac{1}{2 K} .
$$

This function clearly ranges from $\frac{1}{2 K}$ to $1-\frac{1}{2 K}$ in increments of $\frac{1}{K}$, and constitutes the output of our algorithm.

\subsection{Related Work}

The idea of using modified data sets in training is not in itself new. In the field of ensemble methods, machine-learning algorithms are run repeatedly on different data sets resampled from the original one, and the votes of the resulting classifiers are used as a basis for classification. The proportions of votes could also of course be regarded as estimates of class probabilities, but this probabilistic interpretation is not necessarily well-founded. These methods differ from our approach in that only the selection of chosen data points varies; the class labels themselves are not changed. To our knowledge, the only other algorithm that changes class labels of the training examples is MetaCost [6], which operates in a slightly different context, that of costsensitive classification. In MetaCost, an ensemble of classifiers is trained using resampled versions of the training set. The training examples are then relabelled with their optimal classifications, given the chosen misclassification costs and votes from the single classifiers. Any classification algorithm can then be trained on this modified set to provide cost-sensitive classification. Thus, another similarity between MetaCost and the algorithm presented here is that both are able to use any hard classification method as a "black box". We shall return to the link between class probability estimation and cost-sensitive classification in connection with the experiments described in Section 3.2.

\section{Experiments}

In this section, we present results from testing our algorithm on two problems. First, we apply it to a simple illustrative toy problem; the second test employs a data set from the UCI machine learning repository and compares the results achieved with results reported in previous research.

\subsection{Toy Problem Experiment}

As an illustration of our approach, we defined a problem with input space $\left(-\frac{1}{2}, \frac{1}{2}\right) \times\left(-\frac{1}{2}, \frac{1}{2}\right)$ and class probability function $p(y=1 \mid \mathbf{x})=\left(1+\exp \left(9|\mathbf{x}|^{2}\right)\right)^{-1}$. A contour plot of this function is shown in Figure 2a. A training set $D$ of $L=250$ data points $\mathbf{x}$ were drawn with uniform probability over $X$ and labelled with $y$ values in $\{0,1\}$ according to the probability function $p(y=1 \mid \mathbf{x})-$ see Figure $2 b$. 
As a basis for comparison, we first estimated $p(y=1 \mid \mathbf{x})$ by forming a distanceweighted average function using a simple radial basis function (RBF) algorithm, with a Gaussian kernel function

$$
K\left(\left|\mathbf{x}_{i}-\mathbf{x}\right|\right)=\frac{1}{\sqrt{2 \pi} \sigma} \exp \left(-\frac{\left|\mathbf{x}_{i}-\mathbf{x}\right|^{2}}{2 \sigma^{2}}\right)
$$

around each data point $\left(\mathbf{x}_{i}, y_{i}\right)$. This yields the estimate

$$
\hat{p}_{R B F}(y=1 \mid \mathbf{x})=\sum_{i=1}^{L} y_{i} K\left(\left|\mathbf{x}_{i}-\mathbf{x}\right|\right) / \sum_{i=1}^{L} K\left(\left|\mathbf{x}_{i}-\mathbf{x}\right|\right) .
$$

Based on some initial experiments, we set $\sigma=0.15$; Figure $2 \mathrm{c}$ shows the resulting contour plot of $\hat{p}_{R B F}(y=1 \mid \mathbf{x})$.

Next, we estimated the conditional class probability function using only hard classifiers in combination with the algorithm we have described. We used the classification algorithm that results from thresholding $\hat{p}_{R B F}(y=1 \mid \mathbf{x})$ at $\frac{1}{2}$. When run on the original data set $D$, the result is clearly a hard classifier with a decision boundary following the 0.5 contour in Figure 2c.

We partitioned the interval $(0,1)$ into $K=50$ parts, so that $q_{k}=k / 50$ for $k=0,1, \ldots, 50$, and ran the algorithm as described in Section 2.3. That is, for each $q_{k}, k=1,2, \ldots, 49$, we created a modified data set, trained a hard classifier on this modified set, and used Equation (1) as an estimate $\hat{p}_{H C}(y=1 \mid \mathbf{x})$ of the conditional class probabilities. Figure $2 \mathrm{~d}$ shows a contour plot of the results.

A comparison to Figure 2c shows that our algorithm yields similar results, apart from being less smooth - this latter property is not surprising, given the algorithm's discrete and stochastic nature. A more quantitative assessment of the performance of the two algorithms can be gained from estimating the expected negative loglikelihood, according to the probability estimate, of a new data point drawn from $p(\mathbf{x})$ and $p(y=1 \mid \mathbf{x})$. This quantity was estimated using a uniform grid of $51 \times 51$ points over the input space in the following way:

$$
-E[L L(\hat{p})]=-\frac{1}{51^{2}} \sum_{i=-25}^{25} \sum_{j=-25}^{25} \sum_{c=0}^{1} p\left(y=c \mid \mathbf{x}=\left(\frac{i}{50}, \frac{j}{50}\right)\right) \ln \hat{p}\left(y=c \mid \mathbf{x}=\left(\frac{i}{50}, \frac{j}{50}\right)\right) .
$$

Table 1. Expected negative log-likelihood of a new data point according to each model

\begin{tabular}{|l|c|}
\hline$\hat{p}$ & $-E[L L(\hat{p})]$ \\
\hline$p$ & 0.460 \\
\hline$\hat{p}_{R B F}$ & 0.481 \\
\hline$\hat{p}_{H C}$ & 0.482 \\
\hline
\end{tabular}



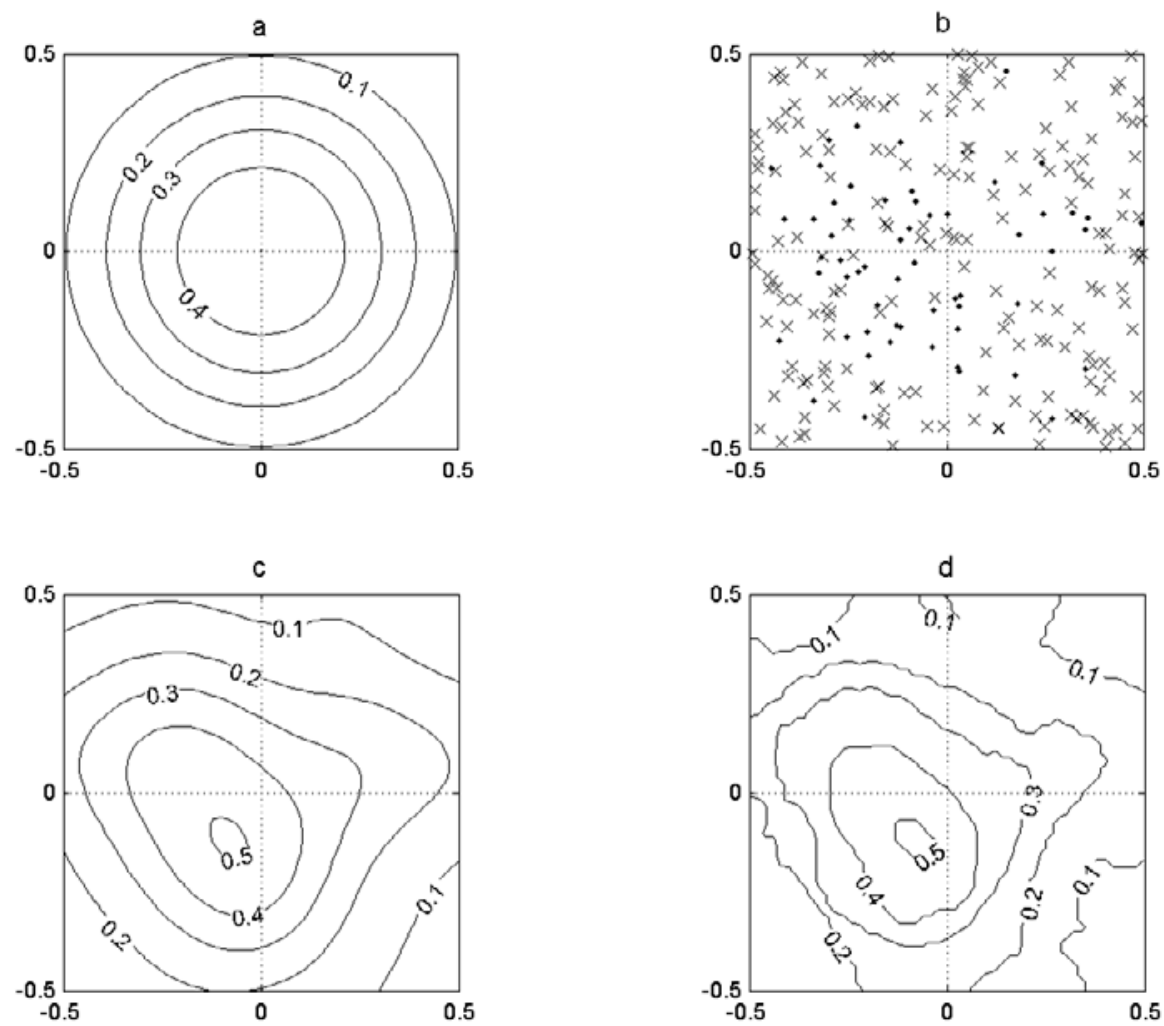

Fig. 2. Experiments with the toy problem. a) Contours of the true conditional class probability function. b) Randomly generated training data; dots represent positive and crosses negative examples. c) RBF regression estimate of the probability function. d) Estimate using modified data sets and hard RBF classifiers

The results for $\hat{p}_{R B F}$ and $\hat{p}_{H C}$, as well as for the true function $p$, are given in Table 1; this confirms that the two algorithms have similar performance.

\subsection{Experiment on the UCI Adult Benchmark}

As a more challenging test for the algorithm, we used the Adult data set from the UCI machine learning repository [7]. Each data point $\mathbf{x}$ here consists of fourteen values (six numeric and eight categorical) taken from a census form for a household; the class label indicates whether the household's yearly income was greater or smaller than USD 50,000. This data set, comprising 32,561 training examples and 16,281 test examples, was used in order to enable direct comparison to the work of Platt [2], in which he presents a method where the outputs of a support vector machine (SVM) are mapped to probabilities by fitting a logistic function. Platt's paper compares the negative log-likelihoods of the test data (held out during training) given the conditional class probability estimates resulting from this approach and from a regularized-likelihood (RL) kernel method [3,4]. We evaluate our algorithm by 
comparing its performance to these reported results, using the same hold-out set and criterion.

For classification, we downloaded the decision tree classification program CRUISE version 1.09 [8] over the Internet. The algorithm used in CRUISE is described in [9]. The motivations for choosing this software package were that it was a) readily available in executable form for the Windows NT platform, and b) free. The point of requiring an executable classification program was that the experiments would, if successful, support our claim that software for hard classification may be used for conditional class probability estimation without modification.

Again, we set $K=50$, and for each $k$ made a modified version of the training data set as described above. CRUISE was then run on each of these sets, where, for simplicity, the default values were used for all parameters except one. The exception was the number of cross-validation folds that CRUISE uses for pruning after building the full decision tree; this parameter was set to 2 rather than the default of 10 in order to reduce running time.

The performance was evaluated by calculating the negative log-likelihood for the hold-out test data set $T$ according to the resulting estimate of the probability function:

$$
-L L(\hat{p})=-\sum_{\left(\mathbf{x}_{i}, y_{i}\right) \in T} \ln \hat{p}\left(y=y_{i} \mid \mathbf{x}_{i}\right) .
$$

Table 2 shows the results, averaged over five runs, alongside those reported in [2], and also gives the equivalent, but more intuitive, geometric mean of the predicted likelihoods of the test examples:

$$
\bar{L}(\hat{p})=\exp \frac{L L(\hat{p})}{|T|}=\left(\prod_{\left(\mathbf{x}_{i}, y_{i}\right) \in T} \hat{p}\left(y=y_{i} \mid \mathbf{x}_{i}\right)\right)^{\frac{1}{|T|}} .
$$

Table 2. Negative log-likelihoods and equivalent geometric means of predicted likelihood for three methods, using the test data in the UCI Adult data set

\begin{tabular}{|l|c|c|}
\hline Algorithm & $-L L(\hat{p})$ & $\bar{L}(\hat{p})$ \\
\hline SVM + logistic & 5323 & 0.721 \\
\hline $\begin{array}{l}\text { RL kernel } \\
\text { method }\end{array}$ & 5288 & 0.723 \\
\hline Hard classifiers & 5394 & 0.718 \\
\hline
\end{tabular}

In this experiment, our algorithm shows slightly poorer performance than the other two methods. However, those methods were specifically designed for this kind of problem, and also employed some preprocessing of the input data representation [2]. We simply applied a ready-made hard classification algorithm - with no data preprocessing and hardly any parameter tunin $1-$ to stochastically modified data sets.

\footnotetext{
${ }^{1}$ The one exception - decreasing the number of cross-validation folds used by CRUISE for tree pruning - should, if anything, probably worsen the performance of the method.
} 
Seen in this light, we consider our results to be remarkably close to those previously obtained.

We have mentioned cost-sensitive classification as one context where estimates of class probabilities are useful. This relationship can also be seen the other way - a set of cost-sensitive classifiers can be used for estimating class probabilities. To see this, consider the case when the cost of classifying a negative example as positive is 1 , while the cost of misclassifying positive examples is $k$. Classifying an example as positive then means that the expected cost of this choice is less than the expected cost of a negative classification, that is, $p(y=0 \mid \mathbf{x}) \cdot 1$ is less than $p(y=1 \mid \mathbf{x}) \cdot k$, or $p(y=1 \mid \mathbf{x})>1 /(1-k)$. Thus, the contour for the probability level $p(y=1 \mid \mathbf{x})=q$ can be estimated by running a cost-sensitive classifier (again as a black box), setting the cost of misclassifying positive examples to $k=(1-q) / q$. Since our chosen hard classifier supports cost-sensitive classification, we also compared our algorithm with the results achieved when estimating the same 49 probability levels by this method, using the same way of estimating the final probability estimates. The result for the cost-sensitive estimation was $-L L(\hat{p})=5716$, which is clearly inferior to the results achieved by our algorithm.

\section{Discussion}

In the informal exposition in Section 2, we considered the decision boundary of a hard classifier as an estimate of the contour in input space where $p(y=1 \mid \mathbf{x})=\frac{1}{2}$. In fact, this view is the basis of the motivation for our algorithm. However, although it is clearly always optimal that a given single data point $\mathbf{x}$ is classified as positive if and only if $p(y=1 \mid \mathbf{x}) \geq \frac{1}{2}$, the assumption that a classifier will show this behaviour over the whole input space does not necessarily hold, even given unlimited training data. The reason for this lies in the inductive bias of any practical classification algorithm a given algorithm is not able to output an arbitrary classification function $c: X \rightarrow\{0,1\}$, but has a limited hypothesis space from which to choose its classifier. This means that the globally best classifier in this hypothesis space, according to classification accuracy, may not classify according to $p$ everywhere. Platt [2] shows, for example, that the decision boundary of a support vector machine trained on the UCI data set used here does not estimate the $p=\frac{1}{2}$ contour well.

This means that although we do not need to do any modification to a classification algorithm in order to apply it the way we have described, it is important that we know something about the relationship between its classification properties and class probabilities. This point of view sheds some light on the good results obtained here using the CRUISE software - like most other classification algorithms based on decision trees, CRUISE assigns to each of its leaf nodes the class to which the majority of the node's examples belongs. When we consider that the set of tests leading to a given leaf node in effect describes a region in input space, it becomes 
clear that this way of assigning class labels makes the resulting decision boundary approximate the $p=\frac{1}{2}$ contour.

A natural objection to our approach is that it may seem unappealing to introduce more noise into an already noisy data set by modifying the training examples. Our answer to this is that the notion of "noise" is really not the correct one in this probabilistic setting. Unlike in many regression problems, where the observations of the function to be learnt consist of the true values with an added noise component, the data in our setting are simply the natural realisations of an underlying Bernoulli probability function. Thus, flipping the labels in a consistent way should be seen as merely shifting the probability levels of the function, rather than introducing more noise. These considerations also give a hint about which problems the algorithm is likely to solve well. Being based on shifting the probability levels in the instance space, it thus implicitly assumes that such a probability-based model is natural for the problem at hand. Consequently, the method should perform best if the problem is indeed truly probabilistic in nature.

The value of our algorithm lies in the fact that it makes classification algorithms applicable to a new class of problems. This approach may thus be useful if a classification algorithm suited to the problem at hand is readily available, but a conditional probability estimation algorithm is not. If the hypothesis representation of the chosen classification algorithm is easily understandable to humans - as is the case for decision trees, for example - this method also has another advantage, namely that it yields descriptions of the regions of input space where the probability of the positive class is greater than a given level.

The main practical disadvantage of our method is that it is somewhat expensive in terms of runtime - for each probability level, a new data set must be created and the classification algorithm run on this set. On the other hand, the user is free to choose an appropriate trade-off between runtime efficiency and the resolution of the estimating function, by selecting the number of probability levels to estimate. Another disadvantage is that it does not naturally generalize to multi-class problems. Of course, the process can be repeated for each class, but it is then unlikely that the estimated probabilities for the classes sum to one. In the special case where the classes have a natural order, however, the process can be run repeatedly by considering the upper $n$ classes as positive examples, with $n$ ranging from 1 to the number of classes minus one, each run yielding a probability function estimate $p_{n}$. The probability levels for class $m$ can then be estimated by considering the difference between the probability functions $p_{m}$ and $p_{m-1}$ [10]. Even in this case, though, consistent estimates are not guaranteed, due to the stochasticity of the algorithm $p_{m}-p_{m-1}$ may erroneously be negative in parts of the instance space.

\section{Conclusion}

We have described a method for obtaining probabilistic estimates of class membership in classification problems, using only hard classifiers. The algorithm works by generating a succession of data sets from the original one. In each of these sets, a proportion of the examples have their class labels flipped in a way that allows the decision boundary of a hard classifier, trained on the modified data, to be 
interpreted as an estimate of a given probability contour. By collecting the resulting set of hard classifiers, we may form an estimate of the true conditional class probability function. The algorithm has been tested on a toy problem and a problem from the UCI data set repository, with encouraging results.

\section{References}

1. MacKay, D. J. C.: The evidence framework applied to classification networks. Neural Computation 4 (1992) 720-736.

2. Platt, J. C.: Probabilities for SV machines. In: Smola, A.J., Bartlett, P., Schölkopf, B., Schuurmans, D. (eds.): Advances in Large Margin Classifiers, MIT Press (2000) 61-74.

3. Wahba, G.: Multivariate function and operator estimation, based on smoothing splines and reproducing kernels. In: Casdagli, M., Eubank, S. (eds.): Nonlinear Modeling and Forecasting, SFI Studies in the Sciences of Complexity, Proc. Vol. XII, Addison-Wesley (1992) 95-112.

4. Wahba, G.: Support vector machines, reproducing kernel Hilbert spaces and the randomized GACV. In: Schölkopf, B., Burges, C. J. C., Smola, A. J. (eds.): Advances in Kernel Methods - Support Vector Learning, MIT Press (1999) 69-88.

5. Tipping, M.: Sparse Bayesian learning and the relevance vector machine. Journal of Machine Learning Research 1 (2001) 211-244.

6. Domingos, P.: MetaCost: A general method for making classifiers cost-sensitive. In: Proceedings of the 5th International Conference on Knowledge Discovery and Data Mining (KDD-99), ACM Press (1999) 155-164.

7. Blake, C. L., Merz, C. J.: UCI Repository of machine learning databases. Irvine, CA: University of California, Department of Information and Computer Science (1998). URL: http://www.ics.uci.edu/ mlearn/MLRepository.html.

8. Loh, W.-Y.: CRUISE v 1.09 web page. URL: http://www.stat.wisc.edu/ loh/ cruise.html.

9. Kim, H., Loh, W.-Y.: Classification trees with unbiased multiway splits. Journal of the American Statistical Association 96 (2001) 589-604.

10. Frank, E., Hall, M.: A simple approach to ordinal classification. In: De Raedt, L., Flach, P. (eds.): Proceedings of the 12th European Conference on Machine Learning (ECML 2001). Lecture Notes in Artificial Intelligence vol. 2167, Springer (2001) 145-156. 